\title{
Anaplastic astrocytoma cells not detectable on autopsy following long-term temozolomide treatment: A case report
}

\author{
HIROFUMI HIRANO ${ }^{1}$, TAKASHI KAWAHARA ${ }^{2}$, MASAKI NIIRO ${ }^{2}$, HAJIME YONEZAWA ${ }^{1}$, \\ TOMOKO TAKAJYOU $^{1}$, YASUYO OHI ${ }^{3}$, IKUMI KITAZONO ${ }^{3}$, KIYOHIRO SAKAE ${ }^{4}$ and KAZUNORI ARITA ${ }^{1}$ \\ ${ }^{1}$ Department of Neurosurgery, Kagoshima University Graduate School of Medical and Dental Sciences, Kagoshima 890-8520; \\ ${ }^{2}$ Department of Neurosurgery, Imamura Bun-in Hospital, Kagoshima 890-0064; ${ }^{3}$ Department of Molecular \\ and Cellular Pathology, Field of Oncology, Kagoshima University Graduate School of Medical and Dental Sciences, \\ Kagoshima 890-8520; ${ }^{4}$ Department of Pathology, Imamura Bun-in Hospital, Kagoshima 890-0064, Japan
}

Received September 27, 2016; Accepted January 10, 2017

DOI: $10.3892 /$ mco.2017.1160

\begin{abstract}
We herein present an autopsy case of a glioma patient who received long-term treatment with temozolomide (TMZ). The patient, a 35-year-old man with a hypointense tumor of the left frontal lobe, without contrast enhancement following gadolinium (Gd) administration on T1-weighted images, underwent tumor removal surgery, after which the tumor was diagnosed as anaplastic astrocytoma. By the third round of surgery, the tumor had progressed to anaplastic astrocytoma with contrast enhancement following Gd administration, and the patient received $60 \mathrm{~Gy}$ of external beam radiotherapy and nimustine hydrochloride (ACNU)-based chemotherapy. After the fifth tumor removal surgery, TMZ was substituted with ACNU chemotherapy, which suppressed tumor progression. Following the 41st TMZ treatment, hemorrhage was observed in the residual tumor, and the hematoma had been replaced by a hemangioma. The hemangioma and surrounding brain tissue was removed during the sixth surgery. The patient survived for 14 years and 9 months after the initial surgery, but succumbed to hydrocephalus due to bleeding from hemangiomas. The histopathological specimens of the first to the sixth surgeries revealed mutant isocitrate dehydrogenase 1 (IDH1; R132H point mutation) and p53-positive tumor cells, but cells positive for the R132H mutation or p53 could not be detected by immunohistochemistry in the autopsy specimens of the brain after 108 courses of TMZ treatment. Mutant IDH1 (R132H) cells were also not detected in the autopsy specimens of the brain by polymerase chain reaction analysis.
\end{abstract}

Correspondence to: Dr Hirofumi Hirano, Department of Neurosurgery, Kagoshima University Graduate School of Medical and Dental Sciences, 8-35-1 Sakuragaoka, Kagoshima 890-8520, Japan

E-mail: hirahira@m2.kufm.kagoshima-u.ac.jp

Key words: isocitrate dehydrogenase 1, p53, astrocytoma, temozolomide, long-term survival, autopsy

\section{Introduction}

Temozolomide (TMZ) chemotherapy has been found to be effective for malignant glioma. The reported median overall survival (OS) at first relapse for anaplastic astrocytoma and anaplastic mixed oligoastrocytoma was 13.6 months and the adverse events were mild to moderate (1). The overall survival (OS) of patients with newly diagnosed anaplastic astrocytoma treated with 4 cycles of TMZ prior to radiation therapy was 23.5 months (2). We herein report the case of a glioma patient whose tumor had progressed to contrast-enhanced following gadolinium (Gd) administration, and was histopathologically diagnosed as anaplastic astrocytoma harboring the isocitrate dehydrogenase 1 (IDH1) R132H mutation. The protein expression of $\mathrm{O}^{6}$-methylguanine-DNA methyltransferase (MGMT) in the tumor cells was negative. Consecutive TMZ treatment suppressed tumor growth. However, after 108 cycles of TMZ treatment, the patient succumbed to hemorrhage from the hemangiomas. No tumor cells were detected on the post-mortem examination of the brain and the tumor appeared to have regressed following long-term TMZ treatment.

\section{Case report}

A 35-year-old man who experienced a convulsive episode originating in the right side was referred to Kagoshima University Hospital (Kagoshima, Japan). Magnetic resonance imaging (MRI) indicated the presence of a tumor in the left frontal lobe. The hypointense tumor on T1-weighted images did not exhibit contrast enhancement following Gd contrast medium administration (Fig. 1A). The patient underwent the first tumor removal surgery in February, 1999, and the histological diagnosis was anaplastic astrocytoma (Fig. 2, row 1). The retrospective analysis for $1 \mathrm{p} / 19 \mathrm{q}$ status by fluorescence in situ hybridization revealed no co-deletion. A second surgery was performed 2 years and 5 months after the first surgery due to tumor recurrence in the anterior wall of the removal cavity (Fig. 1B) and the histological diagnosis was diffuse astrocytoma (Fig. 2, row 2). At 3 years and 3 months after the first surgery, tumor recurrence was detected as a mass 
displaying contrast enhancement following $\mathrm{Gd}$ administration (Fig. 1C) and the histological diagnosis of the specimens from the third surgery was anaplastic astrocytoma (Fig. 2, row 3). The MIB-1 index of the specimen was $\sim 30 \%$. The patient subsequently underwent radiotherapy (40 Gy of extended-field irradiation and $20 \mathrm{~Gy}$ of local irradiation) and chemotherapy with nimustine hydrochloride (ACNU) $100 \mathrm{mg}$ on day 1 and vincristine $2 \mathrm{mg}$ on days 1, 21 and 42, every 6-8 weeks. After 2 cycles of chemotherapy, a new lesion appeared in the deep frontal lobe (Fig. 1D). The patient underwent a fourth surgery 3 years and 8 months after the first surgery (Fig. 2, row 4). Despite continuing chemotherapy and additional CyberKnife treatment, an invasive tumor extended into the wall of the left lateral ventricle (Fig. 1E) and the patient underwent a fifth tumor removal surgery 4 years and 6 months after the first surgery (Fig. 2, row 5).

At 5 years and 3 months after the first surgery, ACNU was replaced with TMZ for 5 days every 4 weeks, as tumor growth and invasion were not controlled by ACNU treatment. The tumor continued to grow, extending to the right frontal lobe through the corpus callosum after 6 cycles of TMZ treatment; however, the tumor growth decelerated. By the 22nd cycle of TMZ treatment, tumor growth had been arrested. TMZ treatment was continued according to the wishes of the patient and his family. At 8 years and 8 months after the first surgery, the 41 st cycle of TZM treatment was administered and tumor progression was suppressed. This patient's long-term, 41-cycle TZM treatment was previously reported in the Japanese language CP Neurosurgery journal, as case 1 in an article reporting long-term TMZ treatment in two patients with high-grade glioma (3). Prior to the 42 nd TZM treatment, hemorrhage occurred in the residual tumor that displayed contrast enhancement following $\mathrm{Gd}$ administration in the left frontal lobe (Fig. 1F-H). The hemorrhagic lesion was converted to a circumscribed hematoma and the size gradually increased (Fig. 1I-K). The patient underwent a sixth surgery to remove the hemorrhagic lesion 11 years and 3 months after the initial surgery. The circumscribed hematoma (Fig. 3A and B) and surrounding tissue (Fig. 2, row 6) were removed after the 69th TMZ treatment and TMZ treatment was continued after this surgery. Additional new lesions were detected in the right frontal lobe. According to the continuous MRI observation, the T2 hypointense mass in the right frontal lobe was diagnosed as a hemangioma, suspected to have developed post-radiation therapy (Fig. 1L). Several new hemangiomas appeared, but no further lesions indicating glioma recurrence. Eleven years and 7 months after the first surgery, the patient was admitted to another hospital due to deterioration of daily activities under continuing TMZ treatment; he underwent neuroendoscopic surgery 13 years and 8 months after the first surgery to treat the hydrocephalus (Fig. 1M) caused by continuous bleeding into the ventricles from the hemangiomas. During the surgery, a cloudy fluid was observed, with precipitation of the hematoma over the aqueduct. Short-term recovery was achieved by relieving the obstruction of the aqueduct and performing a third ventriculostomy by endoscopic surgery (Fig. 1N), but the patient succumbed to respiratory failure caused by severe hydrocephalus with perpetual hemorrhage from the hemangiomas (14 years and 9 months from the first surgery). An autopsy was performed and the brain tissue was carefully examined.

The TMZ treatment was continued until 3 months prior to the patient's death, for a total of 108 cycles. The initial dosage of TMZ treatment was $150 \mathrm{mg} / \mathrm{m}^{2}$ and was increased to $200 \mathrm{mg} / \mathrm{m}^{2}$ from the second treatment onwards. The mean TMZ dosage administered from the third to the 41st cycle was $175 \mathrm{mg} / \mathrm{m}^{2}$. From the 42 nd cycle onwards, the dose was adjusted to maintain the pretreatment white blood cell count $>2,000 / \mu 1$, and the dose was finally decreased to $\sim 130 \mathrm{mg} / \mathrm{m}^{2}$.

Pathological analysis. Surgically obtained specimens were promptly placed in formaldehyde in the surgical room. The specimens were then transferred to the Pathology laboratory and, after appropriate fixation, embedded in paraffin. During the autopsy, the brain was immediately removed, weighed and measured. Images were captured and the 5 regions of interest were removed. These regions included the left frontal, left and right occipital, right cerebellar and intraventricular masses. These samples, as well as the whole brain, were fixed in formaldehyde. Following fixation, brain specimens were also prepared for microscopic examination (a total of 22 blocks). The specimens were finally embedded in paraffin, cut into $4-\mu \mathrm{m}$ sections and stained with hematoxylin and eosin. The most representative specimens were prepared for immunohistochemistry. The sections were mounted on poly-L-lysine-coated glass slides and stained for IDH1 (DIA H09 R132H, mouse IgG2a; Dianova GmbH, Hamburg, Germany), p53 (DO-7, mouse IgG2b; Leica, Newcastle, UK), MGMT (MT3.1, mouse IgG1; Millipore, CA, USA) and Ki-67 (MIB-1, mouse IgG1; Dako, Glostrup, Denmark). Additional staining with glial fibrillary acidic protein (GFAP; 6F2, mouse IgG1; Dako), CD68 (KP-1, mouse IgG1; Dako) and leukocyte common antigen (CD45; 2B11+PD7/26, mouse IgG1; Dako) was performed to observe reactive changes following hemorrhage. The dilution rates and catalog numbers for all the antibodies were as follows: IDH1 (1:20, DIA-H09), MGMT (1:200, MAB16200), p53 (1:600, NCL-L-p53-DO7), Ki67 (1:50, M7240), GFAP (1:50, M0761), CD68 (1:50, M0814) and CD45 (1:50, M0701). The immunostained specimens were visualized using 3,3'-diaminobenzidine as a substrate and hematoxylin was used as a nuclear counterstain. The immunohistochemistry results are presented in Figs. 2 and 3.

IDH1 detection by quantitative polymerase chain reaction ( $q P C R)$. To confirm the wild-type and $\mathrm{R} 132 \mathrm{H}$ mutation of IDH1, the qPCR procedure was applied. Total RNA was extracted from 50-mg paraffin-embedded specimens from the first surgery, the last surgery and the autopsy, using the NucleoSpin totalRNA FFPE Mini kit (Takara Bio Inc., Kusatsu, Japan) according to the manufacturer's instructions. Each $6 \mu \mathrm{l}$ of extract were used to make cDNA by reverse transcription (ReverTra Ace ${ }^{\circledR}$ qPCR RT Master mix with gDNA Remover, Toyobo Co., Ltd., Osaka, Japan). The aliquot of cDNA $(1.5 \mu \mathrm{g} / \mu \mathrm{l})$ was used as template, and qPCR was conducted (StepOnePlus ${ }^{\mathrm{TM}}$ Real-Time PCR System, Applied Biosystems, USA, CA). The primer sets were as follows: GAPDH: Hs99999905_m1 GAPDH (TaqMan Gene Expression Assays); wild-type IDH1: Hs00001019_rf IDH1_rf (TaqMan Mutation Detection Assays); and mutant 

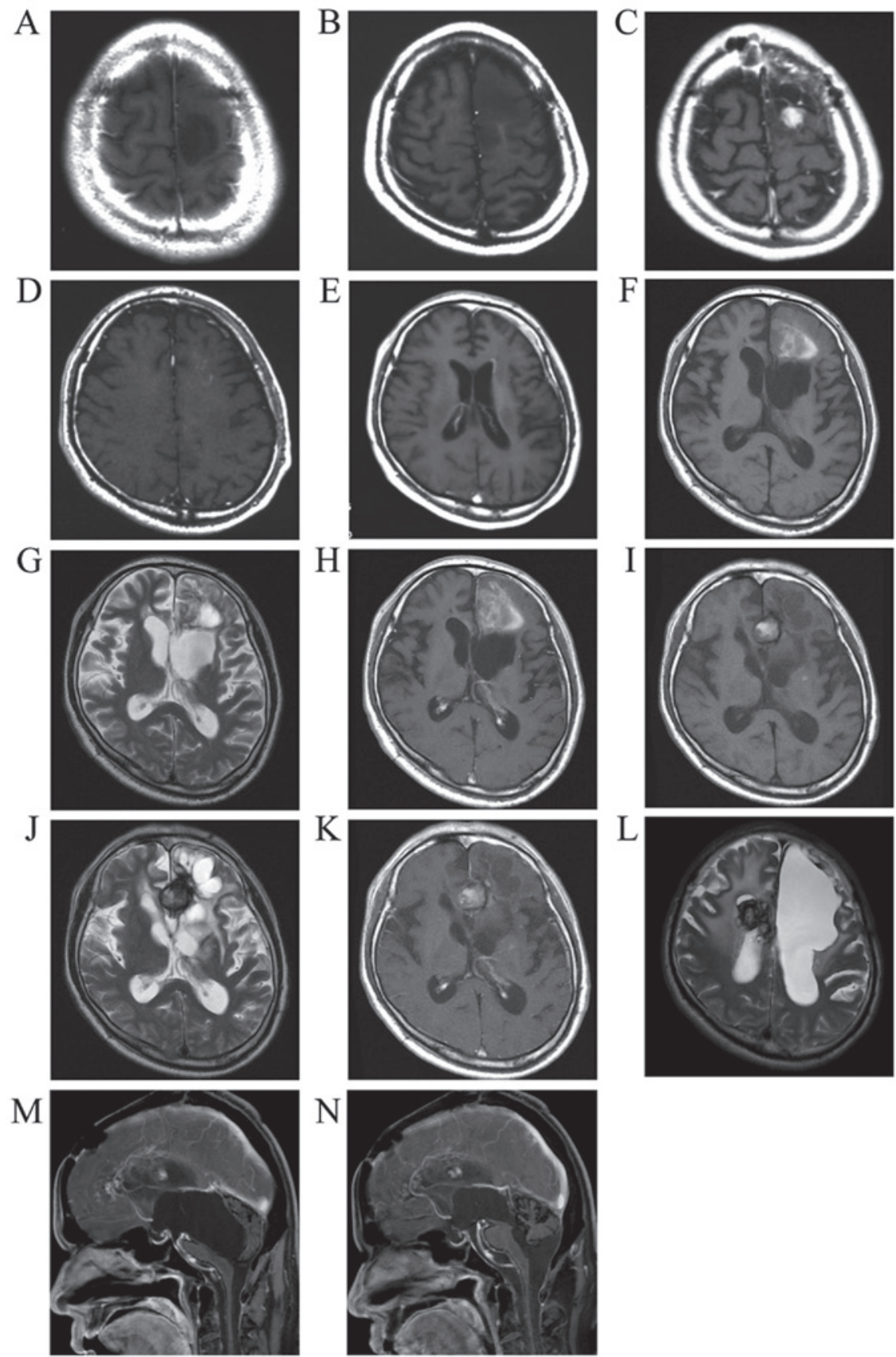

Figure 1. (A) Initial T1-weighted image following gadolinium (Gd) administration (T1Gd). (B) Tumor recurrence prior to the second surgery (T1Gd). (C) Tumor recurrence exhibiting contrast enhancement following Gd administration prior to the third surgery. (D) A new lesion detected in the deep left frontal lobe (T1Gd). (E) Invasion of the tumor extending to the left lateral ventricle. (F) Plain T1-weighted image, (G) T2-weighted image and (H) T1Gd showing bleeding in the tumor of the left frontal lobe. The intratumoral bleeding progressed to a demarcated hematoma as seen on (I) plain T1, (J) T2 and (K) T1Gd images. (L) The same type of demarcated hematoma (hemangioma) occurred in the right hemisphere. (M) Hydrocephalus prior to endoscopic surgery, with a cyst caused by obstruction of the aqueduct protruding into the fourth ventricle. (N) Following endoscopic surgery, the aqueduct was recanalized and the compression of the brain stem was relieved.

IDH1 (R132H): Hs00000981_mu IDH1_28746_mu (TaqMan Mutation Detection Assays).

Histopathological findings. The staining for p53 and IDH1 was positive in all six tumor specimens obtained from each surgery. In the specimens from the sixth surgery, p53 and IDH1 positivity was found in the tissue surrounding the hemangioma. The 21 slides prepared from the specimens obtained from the 5 regions of interest and the 22 slides from the brain specimen were not positive for p53 or IDH1. The tissues from the masses in the lateral ventricles were organized hematomas and consisted of fibroblasts, macrophages and lymphocytes. In the periventricular zone of the left frontal lobe (Fig. 3C-F), non-neoplastic GFAP-positive astrocytes were observed, but these cells were negative for Ki-67, p53 and IDH1. The autopsy specimens were carefully examined, but cells expressing p53 or IDH1 were not detected, leading to the conclusion that the tumor cells had been eliminated from the brain. The MGMT expression was negative in all tumor and autopsy specimens, apart from the blood vessels. 


\section{$\mathrm{H} \& \mathrm{E}$}
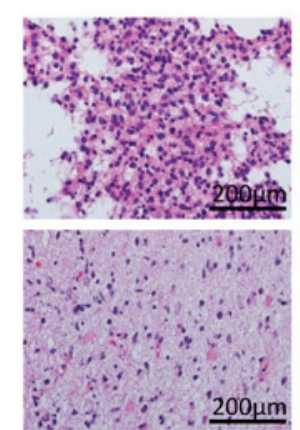
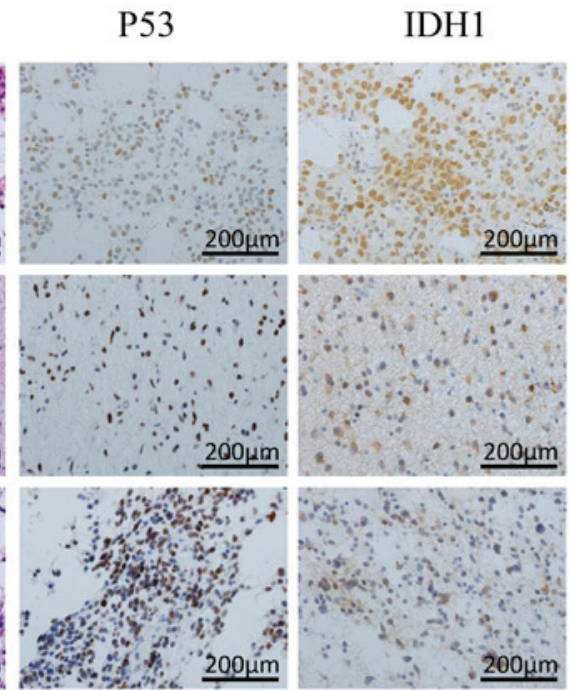

3

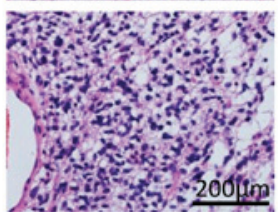

4
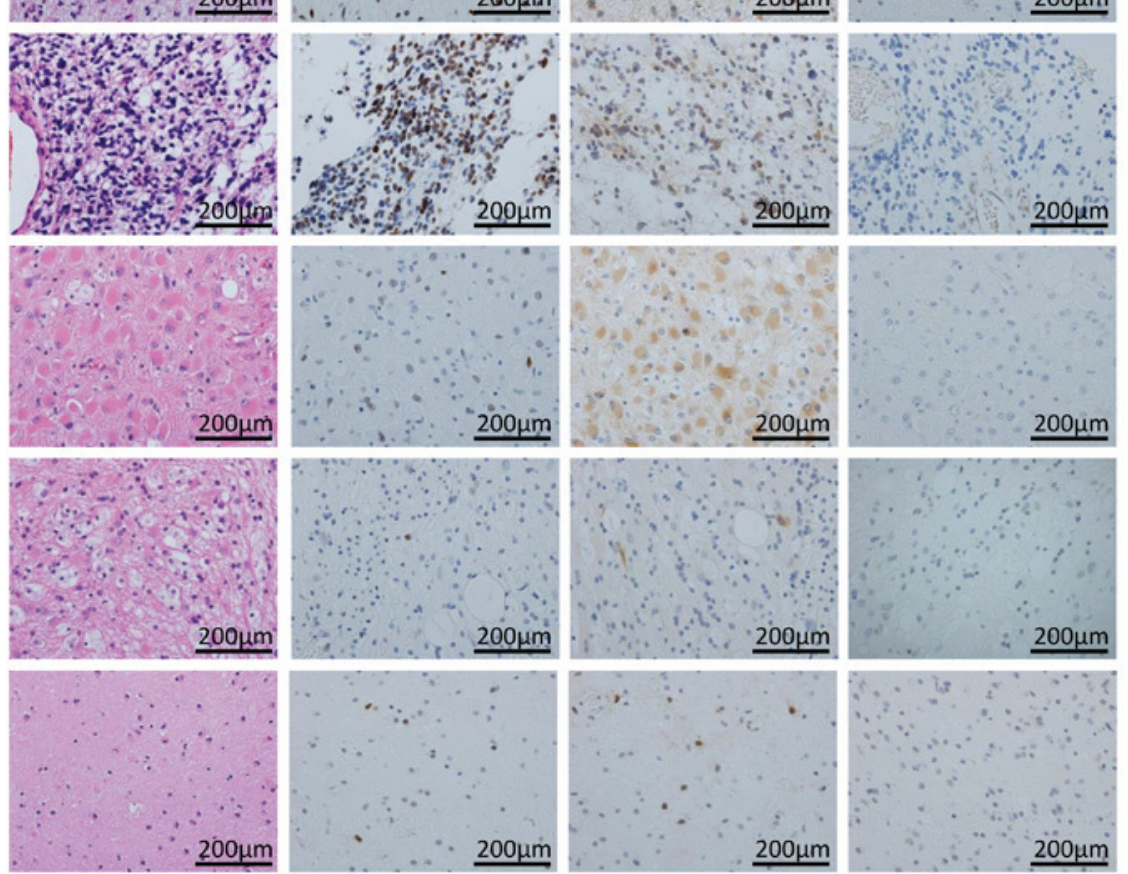

6



Figure 2. Changes in histopathological characteristics from the first surgery (row 1) to the sixth surgery (row 6). p53 and IDH1 were positive in the specimens obtained from all 6 surgeries. H\&E, hematoxylin and eosin; IDH1, isocitrate dehydrogenase 1.

IDH1 message detection by qPCR (Fig. 4). Amplification of cDNA was detected for GAPDH, wild-type IDH1 and mutant IDH1 in the samples from the first and last surgeries. In the autopsy sample, amplification of cDNA for GAPDH and wild-type IDH1 was detected, but that of mutant IDH1 was not. A second PCR was then conducted using the first PCR products as templates in autopsy. However, amplification of mutant IDH1 was not found. This was compatible with the results of immunohistochemistry.

\section{Discussion}

According to recent findings, IDH mutations are a characteristic occurrence in low-grade gliomas $(4,5)$ and considered to be preserved in the glioma lineage. Immunohistochemical identification of the IDH1 and p53 mutations was observed in the specimens from the first surgery in our case; however, the prevalence gradually decreased in the samples obtained through to the sixth surgery, which followed the 69th TMZ treatment, and these mutations were not detected in the autopsy tissue. These results suggested that long-term TMZ treatment eliminated the tumor cells. From an opposing viewpoint, this result indicates that $>69$ courses of TMZ treatment were required to eradicate the tumor cells. The R132H IDH1 mutation is found in $\sim 90 \%$ of all IDH1 mutations (5). The antibody for $\mathrm{R} 132 \mathrm{H}$ is an excellent marker, and Capper et al emphasized its superiority compared with other established antibodies for the differentiation of neoplastic from reactive cells in grade II and III gliomas, allowing for the identification of tumor cells in post-therapy specimens with extensive reactive changes (6). To reconfirm the results of immunohistochemistry, mRNA analysis was performed and the qPCR results established the reliability of immunohistochemistry. Thus, this antibody was found to be a useful and reliable marker for the histopathological detection of tumor cells.

The median OS for the first relapse of anaplastic astrocytoma and anaplastic mixed oligoastrocytoma is 13.6 months, with a response rate of $35 \%$ according to Yung et al (1). The patients with anaplastic astrocytoma reported by Gilbert et al exhibited a good response rate of 39\% (2). A retrospective multicenter study by the Central Nervous System Study Group (Italian Association of Radiation Oncology) reported a median OS of 20.6 months for anaplastic astrocytoma treated by radiotherapy and TMZ, with a survival rate at 4 years of $28.6 \%$ (7). Long-term TMZ treatment is feasible for glioma patients (8) and a case with 85 cycles of TMZ at normal dosing levels was 

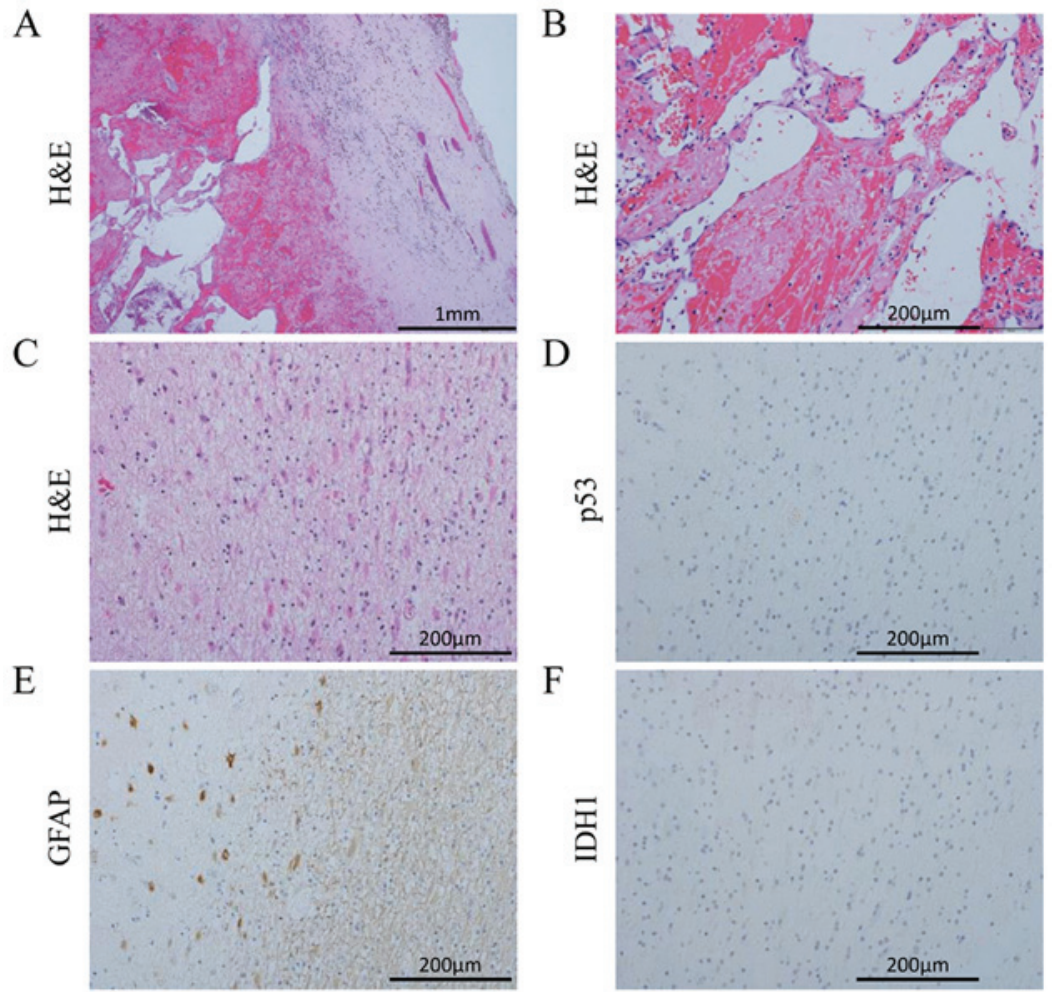

Figure 3. Hemangioma sample obtained during the sixth surgery exhibiting (A) a connective tissue capsule and (B) sinusoidal vessels. In the autopsy specimens of the left frontal periventricular zone, GFAP-positive astrocytes were detected, but the cells were negative for p53 and IDH1 (C-F). H\&E, hematoxylin and eosin; IDH1, isocitrate dehydrogenase 1; GFAP, glial fibrillary acidic protein.

Wild IDH
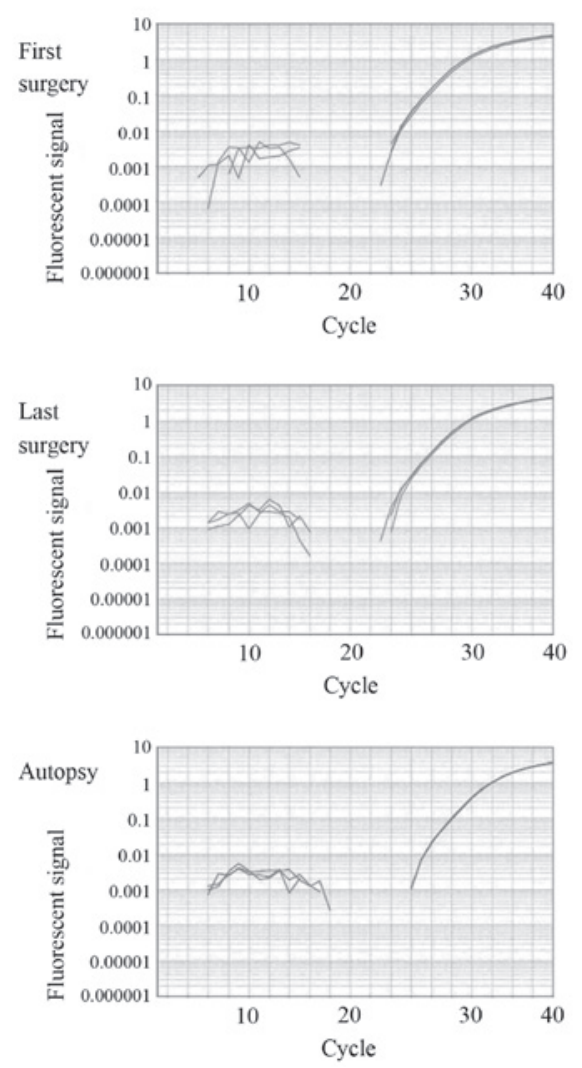

$\mathrm{R} 132 \mathrm{H}$
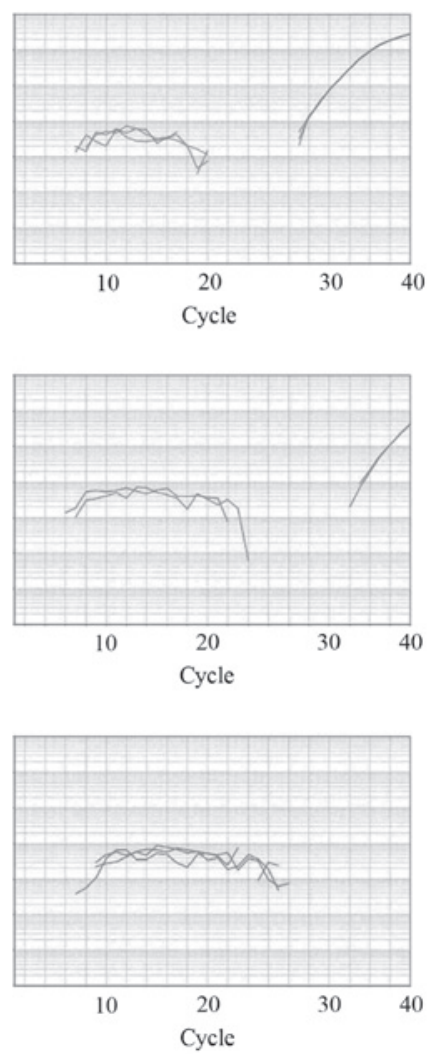

Figure 4. Results of quantitative polymerase chain reaction: Wild-type IDH mRNA was clearly detected in the specimens of the first and last (sixth) surgeries, and in the autopsy specimens (left column). The mutated IDH1 mRNA was consistently detected from the first surgical specimen onwards. The amplification was marginal but detectable in the last surgical specimen. However, in the autopsy specimen, mutated IDH mRNA could not be detected (right column, bottom). 
previously reported (9). In our patient, the dose was reduced to maintain regular treatment, with a total of $108 \mathrm{TMZ}$ cycles administered. The IDH1 mutation is a good prognostic factor in gliomas (10) and, in recent chemoradiotherapy treatments, its presence was associated with prolonged survival time of patients with malignant glioma, even those with glioblastoma (11).

However, long-term survivors may suffer from additional treatment-induced complications. Delayed neurotoxicity by cranial irradiation includes disorders of cognitive function, brain atrophy, radiation necrosis, neoplasms and vascular accidents. Cavernous malformation are frequently reported following radiation therapy in pediatric patients with brain tumors, mostly those with medulloblastoma (12-16). The cumulative incidence of cavernous angioma treated for medulloblastoma was 5.6, 14 and 43\%, at 3, 5 and 10 years, respectively (17). Recent reports have identified that a small number of cavernous angiomas occur after treatment for astrocytic tumors $(18,19)$. This may be attributed to the increased number of long-term astrocytic tumor survivors.

Summarizing the treatment course of our patient, long-term TMZ treatment achieved elimination of tumor cells, although the patient ultimately succumbed to the complications of a cavernous angioma likely induced by radiotherapy. It is currently considered that it is important to remove angiomas during the early stages, in order to prevent continuous bleeding, as recent treatments may significantly prolong the survival of glioma patients.

This retrospective case report conformed to the regulations of the Ethics Committee of Kagoshima University Hospital (reference number 22-50, 'A study of relationship between prognosis and neuroimaging and pathologic findings in brain tumor') and was conducted in accordance with the ethical standards laid down in the 1964 Declaration of Helsinki.

\section{Acknowledgements}

The authors are indebted to the patient who donated his body to glioma research in his living will and grateful to his family who supported his wishes and honored his will. Kazunori Arita has received a Health and Labour Sciences Research Grant from the Ministry of Health, Labour and Welfare of Japan, and the Kiban Research Grant C from the Ministry of Education, Culture, Sports, Science and Technology of Japan. Hirofumi Hirano has received the Kiban Research Grant C from the Ministry of Education, Culture, Sports, Science and Technology of Japan.

\section{References}

1. Yung WK, Prados MD, Yaya-Tur R, Rosenfeld SS, Brada M, Friedman HS, Albright R, Olson J, Chang SM, O'Neill AM, et al: Multicenter phase II trial of temozolomide in patients with anaplastic astrocytoma or anaplastic oligoastrocytoma at first relapse. Temodal brain tumor group. J Clin Oncol 17: 2762-2771, 1999.
2. Gilbert MR, Friedman HS, Kuttesch JF, Prados MD, Olson JJ, Reaman GH and Zaknoen SL: A phase II study of temozolomide in patients with newly diagnosed supratentorial malignant glioma before radiation therapy. Neuro Oncol 4: 261-267, 2002.

3. Hirano H, Yunoue S and Arita K: Long-term temozolomide treatment in two patients with high-grade glioma (in Japanese with English abstract). CP Neurosurgery 18: 886-892, 2008.

4. Yan H, Parsons DW, Jin G, McLendon R, Rasheed BA, Yuan W, Kos I, Batinic-Haberle I, Jones S, Riggins GJ, et al: IDH1 and IDH2 mutations in gliomas. N Engl J Med 360: 765-773, 2009.

5. Arita H, Narita Y, Yoshida A, Hashimoto N, Yoshimine T and Ichimura K: IDH1/2 mutation detection in gliomas. Brain Tumor Pathol 32: 79-89, 2015.

6. Capper D, Sahm F, Hartmann C, Meyermann R, von Deimling A and Schittenhelm J: Application of mutant IDH1 antibody to differentiate diffuse glioma from nonneoplastic central nervous system lesions and therapy-induced changes. Am J Surg Pathol 34: 1199-1204, 2010.

7. Scoccianti S, Magrini SM, Ricardi U, Detti B, Krengli M, Parisi S, Bertoni F, Sotti G, Cipressi S, Tombolini V, et al: Radiotherapy and temozolomide in anaplastic astrocytoma: A retrospective multicenter study by the central nervous system study group of AIRO (Italian Association of Radiation Oncology). Neuro Oncol 14: 798-807, 2012.

8. Hau P, Koch D, Hundsberger T, Marg E, Bauer B, Rudolph R, Rauch M, Brenner A, Rieckmann P, Schuth J, et al: Safety and feasibility of long-term temozolomide treatment in patients with high-grade glioma. Neurology 68: 688-690, 2007.

9. Mannas JP, Lightner DD, Defrates SR, Pittman T and Villano JL: Long-term treatment with temozolomide in malignant glioma. J Clin Neurosci 21: 121-123, 2014.

10. Takano S, Kato Y, Yamamoto T, Kaneko MK, Ishikawa E, Tsujimoto Y, Matsuda M, Nakai K, Yanagiya R, Morita S, et al: Immunohistochemical detection of IDH1 mutation, p53, and internexin as prognostic factors of glial tumors. J Neurooncol 108: 361-373, 2012.

11. Kawano H, Hirano H, Yonezawa H, Yunoue S, Yatsushiro K, Ogita M, Hiraki Y,Uchida H,Habu M, Fujio S, et al: Improvement in treatment results of glioblastoma over the last three decades and beneficial factors. Br J Neurosurg 29: 206-212, 2015.

12. Novelli PM, Reigel DH, Langham Gleason P and Yunis E: Multiple cavernous angiomas after high-dose whole-brain radiation therapy. Pediatr Neurosurg 26: 322-325, 1997.

13. Maeder P, Gudinchet F, Meuli R and de Tribolet N: Development of a cavernous malformation of the brain. AJNR Am J Neuroradiol 19: 1141-1143, 1998.

14. Baumgartner JE, Ater JL, Ha CS, Kuttesch JF, Leeds NE, Fuller GN and Wilson RJ: Pathologically proven cavernous angiomas of the brain following radiation therapy for pediatric brain tumors. Pediatr Neurosurg 39: 201-207, 2003.

15. Aguilera D, Tomita T, Goldman S and Fangusaro J: Incidental resolution of a radiation-induced cavernous hemangioma of the brain following the use of bevacizumab in a child with recurrent medulloblastoma. Pediatr Neurosurg 46: 303-307, 2010.

16. Chourmouzi D, Papadopoulou E, Kontopoulos A and Drevelegas A: Radiation-induced intracranial meningioma and multiple cavernomas. BMJ Case Rep 2013: pii, 2013.

17. Lew SM, Morgan JN, Psaty E, Lefton DR, Allen JC and Abbott R: Cumulative incidence of radiation-induced cavernomas in long-term survivors of medulloblastoma. J Neurosurg 104 (Suppl 2): S103-S107, 2006.

18. Furuse M, Miyatake SI and Kuroiwa T: Cavernous malformation after radiation therapy for astrocytoma in adult patients: Report of 2 cases. Acta Neurochir (Wien) 147: 1097-1101, 2005.

19. Fukushima S, Narita Y, Miyakita Y, Ohno M, Takizawa T, Takusagawa Y, Mori M, Ichimura K, Tsuda H and Shibui S: A case of more than 20 years survival with glioblastoma, and development of cavernous angioma as a delayed complication of radiotherapy. Neuropathology 33: 576-581, 2013. 